\title{
EL CONTINUO LINEAL. INTUICIÓN GEOMÉTRICA O CONSTRUCCIÓN ARITMÉTICA
}

\author{
CARLos ÁlVarez \\ Facultad de Ciencias \\ UNAM
}

Desde la demostración de la independencia de la hipótesis del continuo, en 1963, mediante la utilización de los métodos de forcing, apareció cierta tendencia en matemáticas: el problema del continuo, que había ocupado la atención y el esfuerzo de los matemáticos por más de medio siglo, perdió súbitamente el carácter de problema verdadero al demostrarse su independencia respecto de los axiomas de la teoría de conjuntos.

Después de la prueba de Cohen, el interés de los conjuntistas hacia la hipótesis del continuo desaparecí casi por completo, y, de algún modo, comenzó a predominar la idea de que en torno a esta hipótesis, por tratarse de una proposición independiente, ya no hay nada que decir, nada que añadir y menos aún considerar la posibilidad de buscar alguna estrategia para su demostración. El camino seguido por los conjuntistas desde entonces es paralelo a la vía marcada por la teoría de modelos: de entre modelos de la teoría de Zermelo-Fraenkel, se trata de analizar aquellos en los que esta hipótesis se satisface, para así estudiar las propiedades con las que ésta puede coexistir.

Ante esta actitud, recordemos que Kurt Gödel, poco después de haber probado la consistencia relativa de la hipótesis del continuo con el resto de los axiomas de Zermelo-Fraenkel, vislumbraba ya la posibilidad de su independencia, sin que ello 
cancelara su validez como un problema auténtico, del cual debía ocuparse la teorfa de los conjuntos. Los problemas relativos a la cardinalidad de un conjunto se encuentran en el origen mismo de la teoría de los conjuntos, y el continuo lineal, por su parte, es históricamente el conjunto cuyo conocimiento y análisis motiva el desarrollo de la mayoría de las estrategias conjuntistas. La pregunta sobre la cardinalidad que corresponde al continuo lineal no es, pues, ni ajena ni ilegítima. De nuestra incapacidad para encontrar una respuesta a este problema del interior del sistema de axiomas de Zermelo-Fraenkel, finalizaba Gödel, sólo podemos concluir que se trata de un sistema de axiomas incapacitado para permitirnos conocer algunas de las propiedades esenciales de los conjuntos.

Pero antes de adentrarnos en este dilema acerca de la validez, la permanencia y la pertinencia del problema del continuo conviene señalar que la pregunta sobre la cardinalidad del continuo no es la única que permanece aún sin respuesta. $\mathrm{Al}$ grado que podramos decir que esa entidad matemática que llamamos el continuo lineal está hoy más caracterizada por las preguntas que en torno a ella se pueden plantear sin esperar respuesta, que por su conocimiento pleno. Si bien es cierto que ya hace más de un siglo que consideramos de todo punto descrita y conocida su estructura, también veremos que muchos de los problemas irresolubles en torno al continuo encuentran su raíz precisamente en esa estructura que creemos conocer.

Una primera aproximación a las construcciones clásicas del continuo aritmético de los números reales (nos referimos a las construcciones de Dedekind y Cantor) nos permite observar que se trata de contrucciones equivalentes, aun si los procedimientos específicos de cada una de ellas no nos permiten ver inicialmente esta equivalencia. Dedekind parte de las propiedades algebraicas conocidas del conjunto $Q$ de números racionales: se trata de un campo ordenado - que, por ende, está densamente ordenado - en donde cada elemento $q$ produce un corte de la forma $(A, B)$. Si la propiedad de la densidad del 
campo $Q$ impide la presencia de saltos, es posible detectar lagunas como en el conocido ejemplo de $\sqrt{2}$. La presencia de lagunas en el conjunto no hace sino mostrar que puede existir un corte en el conjunto sin que haya sido producido por elemento alguno. La constatación de que las mismas propiedades de orden se cumplen entre los puntos de una línea recta, expresadas a través de las propiedades de incidencia, llevan a Dedekind a asegurar que se trata de una relación de orden equivalente. Pero la relación de orden en los puntos de la línea recta cumple, además, con la propiedad de la continuidad: dado un corte $(X, Y)$ en la línea recta, éste es producido por un único punto $x$ de la misma. Sobre la naturaleza de esta propiedad Dedekind diría que:

La aceptación de esta propiedad de la línea recta no es más que un axioma por medio del cual atribuimos a la línea su continuidad. $^{1}$

La "creación" de los números irracionales obedece a la intención de completar el sistema de números racionales de modo que el sistema de números ampliado posea la misma propiedad de continuidad que el conjunto de puntos de una línea recta. Dedekind asume así la propiedad de la continuidad para el conjunto de puntos de la línea recta y define el conjunto de números reales como aquel que se obtiene a partir de un procedimiento de completación equivalente al que ya existe en la línea; es decir, mediante la identificación de este sistema con la totalidad de los cortes, existentes en el conjunto Q. A pesar de ello, Dedekind asegura que su método establece un fundamento autónomo, y propiamente aritmético, para la teoría de los números reales, ya que éste difiere de los procedimientos anteriores que identificaban a todo número real con la medida de una cierta magnitud geométrica extendida linealmente. Así, para Dedekind, parece dibujarse un dominio propiamente

1 R. Dedekind, Stetigkeiten und irrationale Zahlen. 
aritmético desde el momento en que las nociones de medida y de magnitud geométrica son desplazadas de la definición de los números; a pesar de que el principio de continuidad sea asumido axiomáticamente, por ser indemostrable, en el dominio de la geometría y transcrito como definición al dominio de la aritmética.

En su polémica con Lipschitz respecto a la originalidad de su método, Dedekind insiste en que ningún axioma o postulado euclideano puede dar cuenta del principio de continuidad, tal y como su principio lo establece. A fin de cuentas el argumento de Dedekind consiste en afirmar que, a pesar de los postulados enunciados en los libros V y X de Los elementos, el principio de continuidad está totalmente ausente del dominio de la aritmética y que, por lo tanto, ningún pasaje de Los elementos puede tomarse como inspirador de una teoría de los números irracionales y de los números reales. En otra palabras, si bien es cierto que para que Dedekind puede derivarse de los postulados euclideanos un principio - que puede ser una aceptación tácita - de la continuidad, su traducción geométrica, expresada por la relación de razón entre dos números, no rebasa el dominio de los números racionales y deja incompleto el tratamiento de los números irracionales cuya introducción es posible mediante el principio de continuidad.

El método de completación de Cantor, por su parte, define a los números reales a partir de la propiedad de convergencia dada originalmente por Cauchy. Cantor define a una serie de números racionales como convergente cuando $\left|a_{n+m}-a_{n}\right|$ deviene infinitamente pequeño, conforme $n$ crece y para cualquier entero positivo $m$. Esta propiedad de la serie expresa que "la serie tiene un límite determinado b". La completación de Cantor consiste justamente en considerar el conjunto de todas las series convergentes de números racionales ( $y$, por ende, el conjunto de números por ellas definidos). Un resultado fundamental en esta construcción es que el conjunto $\mathbb{R}$ de números reales ya no puede extenderse por el mismo procedimiento, aun 
cuando sea importante establecer con precisión si un número se ha obtenido mediante dos, tres o más procesos de convergencia. Una vez completo el sistema de números reales, Cantor procede a comparar sus propiedades con las del conjunto de puntos en una línea recta. ${ }^{2}$ Para Cantor los puntos de una línea se pueden determinar en el momento en que se dispone de una cierta unidad de medida y se fija un punto $O$ de la línea; un punto $x$ se localizará a partir de su distancia al punto fijo $O$. Es posible que la razón de esta distancia con la unidad de medida esté dada por un número racional (en este caso se dice que el punto tiene abscisa racional). Pero si éste no es el caso, será posible encontrar un conjunto infinito $\left\{a_{1}, a_{2}, \ldots a_{n}, \ldots\right\}$ de puntos con abscisa racional, y cuya distancia con el punto $x$ decrece indefinidamente conforme $n$ aumenta (en cuyo caso se dice que la sucesión de puntos se aproxima indefinidamente al punto $x$ ). Si esto es así, la abscisa del punto $x$ es el número real que corresponde a la sucesión convergente de números racionales $\left\{a_{1}, a_{2}, \ldots a_{n}, \ldots\right\}$. Pero la correspondencia deseada entre los números reales y los puntos de una línea recta se establece s6́lo a partir de un axioma. Cantor lo considera así, dado su carácter de indemostrable: a cada magnitud numérica pertenece también un punto de la línea recta. Cantor hace descansar la esencia de la continuidad sobre este axioma, misma que recae, según Dedekind, en el principio que asegura que todo corte $(X, Y)$ en el conjunto (linealmente ordenado) es producido siempre por un único elemento del mismo.

De las construcciones propuestas para el continuo aritmético vemos que en las dos se trata de construir el continuo aritmético a semejanza del continuo geométrico. Dedekind parece inspirarse en la propiedad de continuidad del conjunto de puntos de la línea recta. Esta propiedad del continuo geométrico sólo ha sido descrita parcialmente mediante el axioma de Arqui-

2 Cantor remite para esta construcción al libro $\mathrm{X}$ de Las elementas de Euclides. 
medes, según lo sugiere Hilbert, quien en su Grundlagen der Geometrie hace descansar la propiedad de continuidad sobre dos axiomas, el axioma de Arquimedes:

Si $A_{1}$ es un punto arbitrario, situado entre los dos puntos - también arbitrarios- $A$ y $B$, si se aplica, a partir de $A_{1}$, el intervalo $A A_{1}$, se encontrará un punto $A_{2}$ que distará de $A_{1}$ lo mismo que éste de $A$. Si se sigue este procedimiento, siempre se podrá obtener un punto $A_{n}$ sobre la misma línea y tal que $B$ se encuentre $A$ y $A_{n}$;

y el axioma de integrabilidad (también llamado de saturación), meta-axioma que afirma la inextensibilidad del modelo arquimediano.

Por su parte, Cantor considera que la continuidad se apoya sobre el axioma que asegura que a cada número real corresponde siempre un punto de la línea recta. No es difícil mostrar que, dado un elemento del sistema de números reales definido a la manera de Cantor (como límite de una serie convergente de números racionales), la posibilidad de encontrar un punto de la línea recta que le corresponda se puede garantizar mediante el axioma de Arquímedes: dado un número real $x$, al expresarse en fracción binaria (o de manera equivalente, si es un número del intervalo $\left.(0 \ldots 1), x=d c_{i} / 2^{i}, \operatorname{con} c_{i}=0 \circ 1\right)$ se debe demostrar que un punto de un segmento de longitud 1 corresponde al número $x$. Dicho en otras palabras, que será posible encontar una sucesión infinita de intervalos anidados cuya longitud tiende a cero de modo que el punto que definen tendrá como coordenada al número real dado.

En este sentido el axioma enunciado por Cantor, sobre el que hace descansar la propiedad de la continuidad, sería un principio equivalente al axioma arquimediano. La propiedad de completitud del conjunto de números reales estaría garantizada por una construcción que pretende traducir al ámbito aritmético la propiedad de continuidad de la línea recta. Asf, de esta construcción se deduce la propiedad de la intersección no vacía 
para una familia infinita de intervalos (cerrados) anidados, propiedad conocida como el axioma de completitud de Cantor. ${ }^{3}$ Sobre esta equivalencia, F. Gonseth ${ }^{4}$ afirma que el axioma de saturación puede -y debe- ser sustituido por el axioma de completitud de Cantor para poder establecer la correspondencia biunívoca entre el conjunto de números reales y los puntos de una línea recta.

En una vía más cercana al camino axiomático trazado por Hilbert, 0 . Veblen enuncia ${ }^{5}$ un conjunto de axiomas para el continuo lineal que incluye tanto el axioma de densidad como el axioma de continuidad de Dedekind (propiedad del corte), el axioma pseudoarquimediano (que postula la existencia de una sucesión arquimediana para un conjunto infinito y linealmente ordenado) y el axioma de uniformidad (equivalente al principio de Cantor de los intervalos anidados). Del grupo de axiomas de orden, y del de Dedekin, se deducen tanto el principio de Bolzano-Weierstrass (todo conjunto infinito y acotado posee un punto límite) como el de Heine-Borel (dada una familia de intervalos abiertos que cubren al intervalo cerrado [0... 1], siempre es posible encontrar una subcolección finita que sigue cubriendo al intervalo). A partir de este modo de articular las propiedades de un continuo lineal es posible, para Veblen, deducir la propiedad de separabilidad del continuo lineal a partir del postulado pseudoarquimediano y del principio de uniformidad (mediante el principio de Heine-Borel). ${ }^{6}$ Esta concepción del continuo lineal requiere de la propiedad de cerradura; por lo tanto, es equivalente a la caracterización del

3 De hecho, este postulado de Cantor fue utilizado como consecuencia de su definición en la primera prueba de la no numerabilidad del conjunto de números reales.

4 Les fondements des mathématiques, 1924.

5 "Non-metrical Definition of the Linear Continuum", conferencia ante la SMA en diciembre de 1904.

6 Se puede deducir, a partir de estos argumentos, que es la propiedad de uniformidad la que falta en una línea de Souslin. 
continuo (separable y perfecto) dada por Cantor. Huntington, a su vez, ${ }^{7}$ enuncia el axioma del supremo, parafrasis del principio de Bolzano-Weierstrass, como el axioma de continuidad del que deduce el axioma de Dedekind.

Un recuento de las posibles equivalencias - y de las "combinaciones" necesarias - para poder asegurar que un conjunto de axiomas ha caracterizado de manera completa el continuo lineal, arroja siempre la necesidad de sobreponer o conciliar diversos tipos de operaciones: una operación aritmética, subrayada por Dedekind; una operación analítica, subrayada por Cantor, y una operación geométrica vinculada a todas las formas de utilización del principio de Arquímedes. En cualquier caso, sería posible pensar en el viejo sueño cartesiano: la incorporación del continuo al álgebra, una forma de edificar a la ciencia de la medida y del orden mediante la unificación de dos operaciones esenciales: contar y medir. Sueño éste muchas veces avalado por la afirmación de que en el siglo XIX culminó el proceso de "aritmetización del análisis". Pero esta visión idílica encuentra tropiezos desde el primer momento: dada la identificación entre los puntos de una línea y los números reales, el primer problema - la búsqueda de cuya solución articula a toda la teorfa cantoreana de los conjuntos-consiste en responder a la pregunta sobre el número que corresponde al conjunto de puntos de una línea recta. El propio Cantor demostro, en uno de sus primeros textos, que esta operacion de numeración no es posible mendiante el sistema "usual" de números. Toda su obra apunta hacia la constitución de un sistema de numeración idóneo para esta tarea. Dos interrogantes quedan entonces sin respuesta: por un lado, la legitimidad de una operación de numeración transfinita (que ya ha sido puesta en tela de juicio por los “empiristas" Baire y Borel) y, por el

7 "A Set of Postulates for Real Algebra", conferencia ante la SMA en septiembre de 1904. 
otro, la pregunta acerca del valor numérico específico que corresponde al continuo lineal en esta numeración.

Así, el planteamiento de los dos primeros problemas presentados por Hilbert en 1900 explora las posibilidades de esta conciliación entre el continuo geométrico y el continuo aritmético y busca, de hecho, justificar el término continuo lineal, tan común para nosotros.

Primer problema: Averiguar el número cardinal que corresponde al continuo lineal.

Segundo problema: Probar la no contradicción de los axiomas de la aritmética, i.e. las reglas conocidas para el cálculo, junto con el axioma de continuidad. ${ }^{8}$

Una aproximación más cuidadosa al objetivo de conciliar una entidad aritmética con una entidad geométrica nos muestra que ésta no puede depender de una correspondencia entre sus elementos. Como se ha señalado, esta correspondencia se sustenta en la construcción de una de estas entidades, en una propiedad sugerida por la otra, y traducida e incorporada para obtener precisamente esta correspondencia. Si para Hilbert una definición del continuo lineal, así como su existencia misma, dependía de la consistencia de los axiomas que gobiernan las relaciones mutuas entre ciertos objetos, la estrategia seguida para la prueba de la consistencia consistirá en la inversión de la relación señalada: la consistencia de los axiomas que determinan el continuo geométrico depende de la consistencia —absoluta - de los axiomas aritméticos. En esta perspectiva aparecen también muy pronto los problemas. Así, desde la demostración dada por el propio Hilbert de la independencia del axioma de Arquímedes, surge la posibilidad de un continuo aritmético no arquimediano, sustrato mismo de un análisis no-estándar, cuya historia, según algunos, arranca en

${ }^{8}$ David Hilbert, Mathematische Probleme, Congreso Internacional de Matemáticas, París, 1900. 
Leibniz, pero que en la perspectiva del programa de Hilbert se desprende de la prueba de la no categoricidad de los axiomas aritméticos de Löwenheim-Skolem y de la teoría algebraica de los campos ordenados reales de Artin y Schreier, 9 para desembocar en los trabajos de A. Robinson.

Así, en el intento por definir un continuo aritmético no es posible cerrar los ojos ante el hecho de que los axiomas aritméticos son incapaces de englobar categóricamente el axioma de Arquímedes, vale decir, son incapaces de respaldar la operación geométrica descrita por este principio arquimediano: la medición y el principio de congruencia. Al final de este ciclo, la propiedad singular señalada por Dedekind se muestra como una necesidad esencial.

Pero si esta primera confrontación de los axiomas aritméticos ante las operaciones geométricas muestra ya las dificultades inherentes a la pretendida unidad de las operaciones de contar y medir, las dificultades se presentan también en el interior de la teoría axiomática de los conjuntos. No podemos dejar de señalar esta paradoja planteada por la teoría de los conjuntos al pensamiento matemático: si su prehistoria está íntimamente vinculada al problema de las series trigonométricas y a los problemas relativos al desarrollo y a la expresión de una función en una serie tal, este problema se traduce, primero con Riemann y posteriormente con Cantor, en el problema de la caracterización, desde el punto de vista de la medida, de los conjuntos en los cuales dicha representación es válida. En otras palabras, si este periodo de la historia de la teoría de los conjuntos está íntimamente vinculado con el problema de la integración $y$, por ende, con le.s problemas de medición que ésta supone y maneja, el desarrollo ulterior de la teoría de los conjuntos apunta hacia el desarrollo de la operación de numeración y no al de operación de medición. Pero lo más importante de hacer notar es que la hipótesis de la validez universal (es decir para

${ }^{9}$ Cfr. Hourya Sinaceur, Corps et Modèles, París, 1989. 
cualquier conjunto) de esta numeración transfinita, sugerida ya por el propio Cantor y plasmada bajo la forma de axioma por Zermelo, constituye el argumento más sólido para mostrar las limitaciones que tiene la posibilidad de llevar hasta sus últimas consecuencias a la operación de medición. Ésta es la condición en la que se desarrollla, por ejemplo, el modelo de Solovay, ${ }^{10}$ en el que cualquier conjunto de números reales es medible. Si bajo la modalidad euclideana la operación de medición se realizaba sobre un segmento - de ahí su vinculación con la operación de congruencia - ya desde el inicio de la teoría de la integración, y sobre todo con el desarrollo de la teoría de conjuntos, queda claro que la operación de medición rebasa este ámbito primario y requiere para su desarrollo de trascender el horizonte marcado por la operación de congruencia.

Sin embargo, de estas dificultades no podríamos concluir simplemente la imposibilidad de traducir al modo aritmético cierta intuición geométrica. Acerca de los principios asumidos y reconocidos por Cantor y Dedekind sobre el continuo geométrico es necesario subrayar que no son extraf́dos de una intuición primaria; se trata más bien de principios que dependen, desde Euclides hasta Hilbert, de una operación racional enunciada por el principio de Arquímedes y por las reglas de proporcionalidad de Eudoxio, entre otros. Es decir, una vez trascendido el espacio de conciliación dado por la correspondencia entre dos construcciones racionales, lo que debemos explorar es si hay incompatibilidad entre las dos operaciones: la operación geométrica y la operación aritmética.

La operación geométrica de la medición tal y cómo esta se expresa y es posibilitada por el principio de Arquímedes, supone un manejo peculiar entre un segmento y los puntos de una línea recta. Fiel a la relación establecida por Euclides, se parte de dos puntos que determinan a un segmento; una vez

10 R.M. Solovay, A Model Where Every Set of Real Numbers is Lebesque Mesurable. 
fijado éste, cualquier hipótesis acerca de los puntos contenidos en él carece por completo de sentido (a menos que, siguiendo a Zenón, se acepte una partición del segmento). La operación de medición requiere así de dos puntos distintos o, lo que es equivalente, de un segmento cuyos extremos sean estos dos puntos. ${ }^{11}$ Si se parte de los axiomas de Hilbert, la noción de incidencia impide soslayar la posibilidad de que una infinidad de puntos se encuentren -yazcan o incidan-en el segmento, pero la única determinación susceptible es, antes de la idea de medición, la relación de orden. Así, la noción de distancia, que es susceptible de ser cuantificada, se refiere necesariamente a la distancia entre dos puntos; la idea de medida es la misma que la de distancia (se mide la longitud del segmento) y esta operación desplaza a un segundo plano a la noción de orden de incidencia, que da origen a la idea de número en todas las construcciones axiomáticas desde Peano hasta Von Newmann.

Según Hermann Weyl, en su texto Das Kontinuum, la posibilidad - y necesidad - de hablar de un continuo de números reales nos hace creer que los números reales, considerados como elementos de un conjunto, constituyen un continuo; mientras que, bajo esa 6́ptica, los números reales están tan aislados como los números naturales. Lo que constituye un continuo de números no es la colección o totalidad de ellos (eso es lo que las construcciones de Cantor y Dedekind pretenden mostrar), sino una relación que trasciende necesariamente el ámbito de la relación conjunto-elemento:

Un continuo no tiene elementos sino partes, el concepto de punto debe considerarse como idea límite. Un punto es la representación límite de un proceso de división continuada al infinito. ${ }^{12}$

Esta posición, que no puede dejar de recordarnos a Zenón, forma parte de un subsuelo común para las sucesiones de elec-

11 Cfr. la definición 3 del libro 1 .

12 H. Weyl, Uber die neue Grundlegenkrise der Machematik. 
ción que generan el continuo de Brouwer, y también las mónadas del continuo no-estándar de Robinson.

No podemos olvidar las afirmaciones de Wittgenstein, ${ }^{13}$ aunque sobre la filosofía de las matemáticas de este autor se debe ser muy prudente, acerca de la relación entre punto y línea que se desprende de la nociones conjuntistas con las que esta relación se pretende explicar:

La matemática está enteramente contaminada por la forma perniciosa de las expresiones de la teoría de conjuntos. Se dice, por ejemplo, que una recta está formada de puntos. Una recta es una ley y no está formada por nada.

A este respecto el propio Wittgenstein plantea en sus Philosophical Remarks lo que a nuestro juicio constituye la pregunta filosófica central: "ies posible describir el continuo tal y como Cantor y otros lo intentaron?"

Recordemos que en 1895, en una obra de madurez, Cantor había caracterizado al tipo ordinal $i$ del conjunto de números reales del intervalo $[0,1]$ como aquel que cumple las siguientes propiedades:

1. Es no numerable.

2. Es perfecto.

3. Contiene un subconjunto en él denso y numerable (el conjunto $Q$ de números racionales).

De la tercera propiedad se desprende fácilmente que toda colección de intervalos abiertos y ajenos dos a dos es, a lo más, numerable.

En 1920, el primer volumen de la revista matemática polaca Fundamenta Mathematicae publicó en su última sección el siguiente problema (formulado por M. Souslin):

13 Cfr. Philosophical Remarks y Philosophical Grammar. 
Un conjunto ordenado (linealmente), sin saltos ni lagunas y tal que todo conjunto de intervalos no vacíos y ajenos dos a dos es a lo más numerable, ¿es necesariamente un continuo lineal (ordinario)?

La llamada hipótesis de Souslin es la proposición que responde de manera afirmativa a este problema. Por su enunciado, el problema parece enfocado hacia la teoría de los tipos de orden y, en particular, hacia la caracterización del tipo de orden del continuo lineal. La pregunta planteada por Souslin es, así, una pregunta sobre la equivalencia entre la propiedad requerida en el problema, llamada la propiedad de la cadena numerable, y la tercera condición de Cantor, la separabilidad del continuo lineal. Podríamos definir al tipo ordinal $i^{\prime}$ de un conjunto $E$ como aquel que satisface:

1. Es no numerable.

2. Es perfecto.

3. Toda colección de intervalos abiertos y ajenos es, a lo más, numerable.

La hipótesis de Souslin aseguraría que $i=i^{\prime}$.

Una vez planteada esta nueva interrogante, nos encontramos, como en los casos anteriores, ante una proposición indecidible. No sólo en torno a la congruencia y el orden, como actos generadores de las operaciones de contar y medir, sino también en cuanto se refiere a la caracterización del tipo ordinal del continuo; de modo que, nuevamente, es la ausencia de una respuesta precisa lo que caracteriza el continuo lineal.

K. Gödel, en su texto What is Cantor's Continuum Problem?, proponía iniciar la búsqueda de nuevos axiomas que permitieran decidir afirmativa o negativamente la hipótesis de Cantor acerca de la potencia del continuo. Más allá de las interpretaciones "platonistas" sobre este texto -que, vale decir, para los matemáticos nunca serán secundarias - es necesario rescatar la invitación a analizar ciertas propiedades 
como suceptibles no sólo de coexistir con los axiomas de la teoría de conjuntos, sino también de satisfacer la intuición que de estas propiedades se tiene en el horizonte más amplio de nuestro conocimiento matemático. Parece, entonces, que es necesario aclarar de nueva cuenta el conjunto de nociones sobre el que basamos nuestro conocimiento matemático acerca de una entidad que consideramos ya perfectamente analizada, y establecer, como lo proponía Hermann Weyl, sus ámbitos de pertinencia. La potencia del continuo fue presentada por Cantor como el primer invariante conjuntista para los conjuntos continuos y, al mismo tiempo, mediante este desafío a la intuición geométrica establecía el ámbito de reflexión propia de la teoría de los conjuntos. Pero tras el ejemplo del conjunto perfecto y denso en ninguna parte, antítesis misma de un conjunto continuo y nuevo desafío a la intuición, no es posible creer que una cierta cardinalidad o potencia caracteriza de manera exclusiva a los conjuntos continuos. Podemos así aceptar, y en algunos casos rectificar, algunas de las propiedades que Gödel consideraba como "plausibles" dentro de lo que él mismo llamaba "nuestra intuición intelectual". Debemos mencionar, por ejemplo, la propiedad de todo conjunto no numerable de números reales de contener a un subconjunto perfecto. Hasta ahora todos los casos conocidos - conjuntos $B$-medibles, conjuntos analíticos y proyectivos - poseen esta propiedad, que corrobora parcialmente la hipótesis del continuo. Si Gödel consideraba esta propiedad como "antinatural" al asegurar que, aceptando el axioma de elección, debería mostrarse claramente un conjunto de la potencia del continuo sin ningún subconjunto perfecto, no debemos olvidar, pese a lo que establecimos pocas líneas más arriba, que el ser un conjunto perfecto es una condición que Cantor considera necesaria para los conjuntos continuos.

Así, el ciclo histórico iniciado con la "aritmetización del análisis" -ciclo que muchos historiadores consideran como el que marca el nacimiento de la matemática moderna al darse en 
él de manera definitiva la introducción del continuo geométrico al modo aritmético- deja ver un fin, vislumbrado ya por el propio Gödel en su Declaración sobre el análisis no-estándar y caracterizado, sobre todo, por un cúmulo de preguntas sin respuesta. Si un nuevo ciclo histórico se anuncia, los problemas presentados muestran que éste será el de una nueva síntesis de las matemáticas, generada a partir de una nueva aproximación y un nuevo estudio de una de las más antiguas entidades matemáticas: el continuo lineal.

Recibido: 3 de abril de 1991. 


\section{SUMMARY}

The question whether the different constructions for the linear continuum, given in the last century under that movement known as the period of "arithmetisation" of mathematical analysis, are based on new principles, or if they just describe in other words those geometrical properties already described in Books $\mathrm{V}$ and $\mathrm{X}$ of Euclid's Elements. The question whether the algebraic contruction of the real numbers, within the theory of real fields, is able to describe l'essence de la continuité. The question whether the order type of the real numbers system is a categorical description. The question concerning the existence or non existence of other kinds of linear continua, such as Souslin's line. The question of the "deep meaning" (in Gödel's sense) of the continuum hypothesis. All these are questions raised out from this "very intuitive mathematical object", the linear continuum. 